\title{
Atomic vibrations in disordered systems: Comparison of disor- dered diamond lattices and a realistic amorphous silicon model
}

\author{
J. K. Christie*, S. N. Taraskin, and S. R. Elliott \\ Department of Chemistry, University of Cambridge, Lensfield Road, Cambridge, CB2 1EW, UK
}

\begin{abstract}
PACS 61.43.-j
Force-constant and positional disorder have been introduced into diamond lattice models in an attempt to mimic the vibrational properties of a realistic amorphous silicon model. Neither type of disorder is sufficient on its own to mimic the realistic model. By comparing the spectral densities of these models, it is shown that a combination of both disorders is a better representation, but still not completely satisfactory. Topological disorder in these models was investigated by renumbering the atoms and examining the dynamical matrix graphically. The dynamical matrix of the realistic model is similar to that of a positionally-disordered lattice model, implying that the short-range order in both systems is similar.
\end{abstract}

1 Introduction The physics of atomic vibrations in perfect crystals is well understood [1 [2]. In the presence of disorder (e.g. noncrystallinity), many extra phenomena are observed: for example, localization [3], the boson peak (extra modes at low frequencies) [4], and anomalies in the specific heat |5] and thermal conductivity [6]. The microscopic origin of these and other, similar, phenomena have been difficult to deduce. In particular, it would be helpful to know the contributions due to different types of disorder. In this paper, we consider the effects of three types of disorder: force-constant disorder, in which the strengths of the springs between the atoms are disordered, positional disorder, in which the atomic positions are disordered, and topological disorder, in which the network of bonds is disordered. We compare the behaviour of models with force-constant and positional disorders (separately and in combination) to those of a realistic atomic model. A further aim is to see if it is possible to mimic the behaviour of amorphous systems with an appropriately-disordered crystal.

2 Force-constant and positional disorder In this paper, we study 3D diamond-cubic crystal lattices, in which all atoms are connected by springs, and have the same mass. We assume that the displacements from equilibrium are small, and use the harmonic approximation. Hence, we can treat the system classically [1], and describe the vibrations by a Hermitian dynamical matrix $\hat{\mathbf{D}}$. The problem can then be treated in the Hamiltonian formalism [7, 8, 9], with energy $\epsilon=\omega^{2}$, the squared vibrational frequency. We study the effect of disorder on the spectral density: $\hat{\mathbf{A}}(\epsilon)=\langle\delta(\epsilon-\hat{\mathbf{D}})\rangle$, where $\langle\ldots\rangle$ represents configurational averaging. We can represent the spectral-density operator in the crystalline basis $|\mathbf{k}, \beta\rangle$, where $\mathbf{k}$ is the wavevector of a plane wave, and $\beta$ is the polarization [10]. In this case, the spectral density $A_{\mathbf{k} \beta}(\epsilon)$ is:

$$
A_{\mathbf{k} \beta}(\epsilon)=\left\langle\sum_{d}|\langle d \mid \mathbf{k}, \beta\rangle|^{2} \delta\left(\epsilon-\epsilon_{d}\right)\right\rangle,
$$

where $|\langle d \mid \mathbf{k}, \beta\rangle|^{2}$ are the weights of the (disordered) eigenstate $|d\rangle$ (with energy $\epsilon_{d}$ ) in the crystalline eigenstate $|\mathbf{k}, \beta\rangle$. Large ( $10^{5}$ atoms) diamond-cubic crystals have been simulated, with nearest-neighbour

\footnotetext{
* Corresponding author: e-mail: jkc25@cam.ac.uk
} 


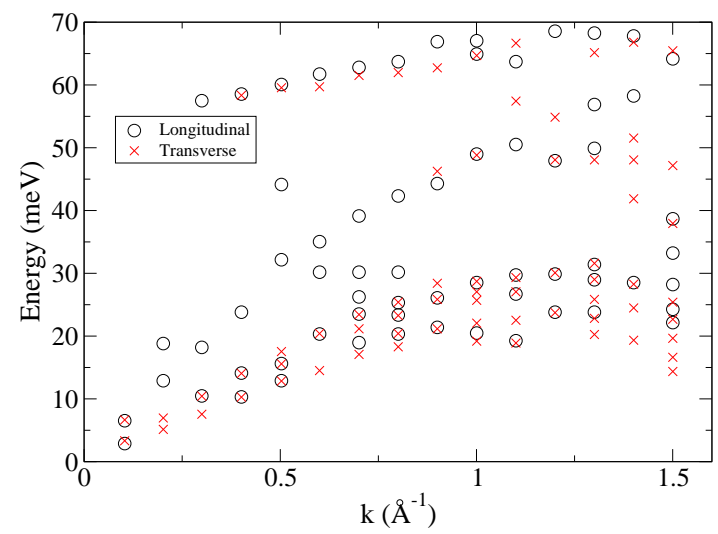

Fig. 1 Longitudinal (circles) and transverse (crosses) dispersion relation for a positionally-disordered diamond lattice with $\sigma=0.1 r_{1}$, where $r_{1}$ is the nearest-neighbour distance. Mixing between polarisations is clear.

spring constant $\kappa_{1}$ and next-nearest-neighbour spring constant $\kappa_{2}$. A ratio of $\kappa_{1} / \kappa_{2}=8$ was chosen, as this gave a vibrational density of states (VDOS) most like that of a crystal with the more realistic modified Stillinger-Weber (mSW) potential [11, 12]. Force-constant disorder was added by randomly taking the spring constants from a uniform distribution of half width $\Delta$, centred on the crystalline spring-constant value. The width $\Delta$ is expressed as a fraction of the crystalline spring-constant value $\kappa$, and this fraction is the same for both spring constants. To avoid mechanical instabilities, widths were limited such that all force constants were positive, i.e. $\Delta \leq \kappa$. Positional disorder was introduced by randomly shifting all atoms by distances, chosen from Gaussian distributions centred on zero with half width $\sigma$, independently in the $x$-, $y$ - and $z$-directions.

Figure 1 shows the dispersion relation for a positionally-disordered diamond lattice with $\sigma=0.1 r_{1}$, where $r_{1}$ is the nearest-neighbour distance. At each value of $k$, the spectral density has been orientationally averaged, and the positions of the peaks are shown in the figure. The dispersion relation shows many of the expected features: three acoustic branches are observed, of which one is longitudinal, and two are transverse. Mixing between the polarisations is seen: the longitudinal spectral density picks up the peaks due to the transverse acoustic (TA) branches. The optic modes are less clearly defined; the peaks due to these modes were broadened considerably by even small amounts of disorder. Nevertheless, the presence of optic branches at high energy in both the transverse and longitudinal spectral densities is observed.

The effect of a low degree of positional disorder on the spectral density is qualitatively very similar to the effect of small force-constant disorder [13]; the peak shifts in position, and broadens. When compared to the spectral density of a realistic 4096-atom model of amorphous silicon, generated with a modified WWW procedure [14 15] and relaxed under the mSW potential, it was observed that neither type of disorder was adequate on its own to reproduce the features of the amorphous model's spectral density.

A combination of these two types of disorder is better able to reproduce the spectral density, as seen in Figure 2] The disordered lattice models are able to mimic the main features of the amorphous model, with peak positions and shapes at least roughly correct, but they often have more peaks (particularly at high $k$ ) than the rather smoother amorphous spectral densities. This is due to the orientational averaging - each of these peaks comes from a different orientation of the k-vector. The fact that these peaks are at different energies suggests that, despite the added disorder, the lattice models are still anisotropic, whereas the amorphous model is isotropic (since the multiple peaks are absent). This implies that a form of disorder needs to be considered that will render the lattice models less anisotropic. 


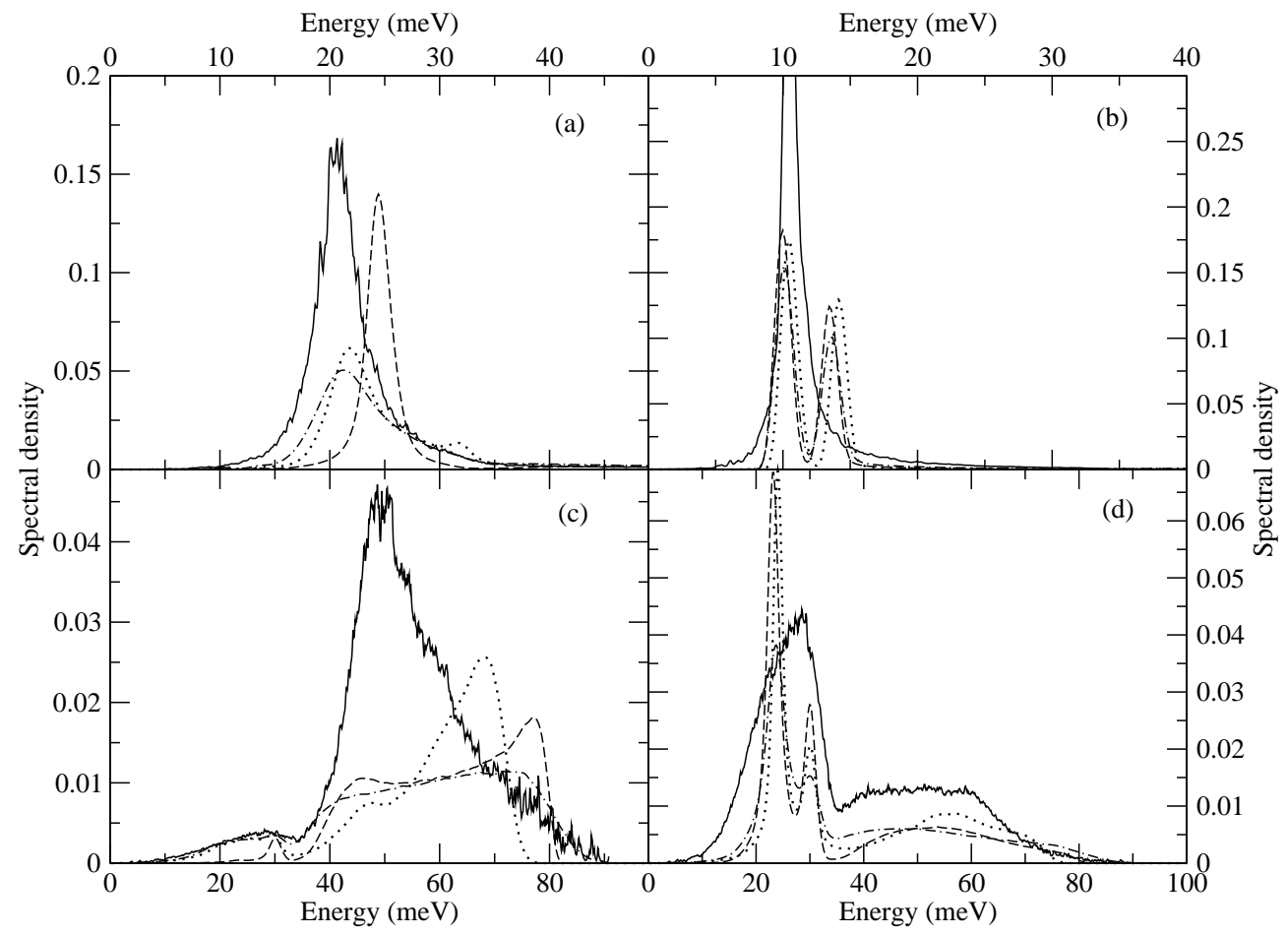

Fig. 2 Orientationally-averaged spectral densities of a realistic 4096-atom amorphous silicon model compared to disordered 125 000-site diamond lattices. Each graph shows the spectral densities of the realistic model (solid line), a lattice model with force-constant disorder $\Delta=0.75 \kappa$ (dashed line), a lattice model with positional disorder $\sigma=0.15 r_{1}$ (dotted line), and a model with both the above force-constant and positional disorders (dot-dashed line). $k=0.401 \AA^{-1}$ ( $k=0.411 \AA^{-1}$ for the realistic model): (a) longitudinal, (b) transverse; $k=1.200 \AA^{-1}$ ( $k=1.197 \AA^{-1}$ for the realistic model): (c) longitudinal, (d) transverse. The value of $k$ is slightly different between the lattice and realistic models due to the necessity of using $\mathbf{k}$-vectors that fit the periodic boundary conditions.

3 Topological disorder Another type of disorder is topological disorder, in which the bond network is disordered such that the ring-size distribution is no longer crystalline. Topological disorder was examined by studying the dynamical matrix. If two atoms $i$ and $j$ interact, then the dynamical matrix elements representing that interaction will be non-zero. We make use of a simple atom renumbering scheme and show the dynamical matrix graphically, to compare the topological disorder present in the realistic model and in positionally-disordered models.

Atoms were renumbered by first dividing the models into small cubes, such that the average occupation was one atom per small cube. The models are cubic, with side $L$. The origin was taken at one corner of the model (say $x, y, z=0$ ), and atoms were numbered by moving up through all the small cubes above it in the positive $z$-direction, numbering the atoms in order of the $z$-coordinate of their position. Once the $z=L$ face of the model was reached, the scheme returned to the $z=0$ face, and moved along one small cube distance in the $y$-direction. As before, atoms were numbered by moving up in the $z$-direction. This process was repeated until the $y=L$ face was reached, when the scheme returned to $y, z=0$ and shifted along one small cube in the $x$-direction, and then repeated the numbering above, until all atoms were numbered. The atom number now corresponds (in some way) to its position. 
(a) \

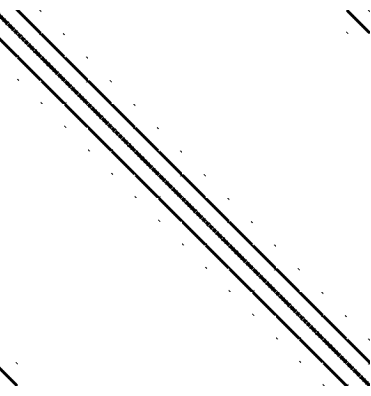

(b)

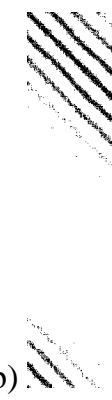

(c) 1

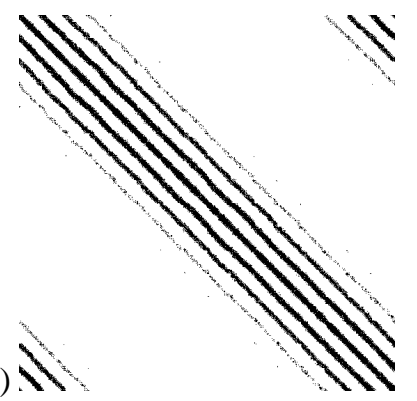

Fig. 3 Graphical representation of the dynamical matrix of: (a) a diamond crystal with nearest- and nextnearest-neighbour interactions; (b) the same crystal positionally disordered with $\sigma=0.6 r_{1}$; (c) a realistic 4096-atom model of amorphous silicon.

In this renumbering scheme, the dynamical matrix of a crystalline diamond lattice with nearest- and next-nearest neighbour interactions is shown in Fig. 3 $\mathrm{k}$. The effect of introducing positional disorder $\left(\sigma=0.6 r_{1}\right)$, into the diamond lattice, is shown in Fig. $3 \mathrm{~b}$. The realistic model of amorphous silicon, with the same renumbering scheme, is shown in Fig. 3k. Remarkably, the dynamical matrix for the realistic model (Fig. 35) looks very similar to that of the positionally-disordered crystal (Fig. 3b). We interpret this as implying that the short-range structural order, responsible for the vibrational behaviour, in the topologically-disordered amorphous model is very close to that in a positionally-disordered crystal.

4 Conclusion Examining the spectral densities of diamond lattice models with added force-constant and positional disorders shows that these disorders are insufficient (either by themselves or in combination) to mimic completely the vibrational behaviour of a realistic model of amorphous silicon. Representing the neighbour list graphically shows that the short-range order, responsible for the vibrational behaviour, in a positionally-disordered diamond lattice is similar to that of a realistic model, and this is an avenue for further research.

Acknowledgements JKC is grateful to the Engineering and Physical Sciences Research Council for financial support. We thank G. T. Barkema for providing us with the atomic co-ordinates of the amorphous model used.

\section{References}

[1] A. A. Maradudin, E. W. Montroll, G. Weiss and I. P. Ipatova, Theory of Lattice Dynamics in the Harmonic Approximation (Academic Press, New York, 1971).

[2] M. T. Dove, Introduction to Lattice Dynamics (Cambridge University Press, 1993).

[3] S. John, H. Sompolinsky and M. J. Stephens, Phys. Rev. B 27, 5592 (1983).

[4] S. N. Taraskin, Y. L. Loh, G. Natarajan and S. R. Elliott, Phys. Rev. Lett. 86, 1255 (2001).

[5] J. L. Feldman, P. B. Allen and S. R. Bickham, Phys. Rev. B 59, 3351 (1999).

[6] R. O. Pohl, X. Liu and E. Thompson, Rev. Mod. Phys. 74, 991 (2002).

[7] R. J. Elliott, J. A. Krumhansl and P. L. Leath, Rev. Mod. Phys. 46, 465 (1974).

[8] H. Ehrenreich and L. Schwarts, Solid State Physics 31, 149 (1976).

[9] E. N. Economou, Green's Functions in Quantum Physics, (Springer, Berlin, 1983), 2nd edition.

[10] S. N. Taraskin and S. R. Elliott, J. Phys.: Condens. Matter 14, 3143 (2002).

[11] F. H. Stillinger and T. A. Weber, Phys. Rev. B 31, 5262 (1985).

[12] R. L. C. Vink, G. T. Barkema, N. Mousseau and W. F. van der Weg, J. Non-Cryst. Solids 282, 248 (2001)

[13] S. N. Taraskin, J. J. Ludlam, G. Natarajan and S. R. Elliott, Philos. Mag. B 82, 197 (2002).

[14] F. Wooten, K. Winer and D. Weaire, Phys. Rev. Lett. 54, 1392 (1985).

[15] G. T. Barkema and N. Mousseau, Phys. Rev. B 62, 4985 (2000). 